\title{
EXPERIENCE OF ANTIRETROVIRAL TREATMENT IN GEORGIA
}

\author{
Tengiz Tsertsvadze ${ }^{1,2}$, Natalia Bolokadze ${ }^{1}$, Nino Gochitashvili', Lali Sharvadze ${ }^{1,2}$, Otar Chokoshvili', Natia Dvali', \\ Amiran Gamkrelidze 3 , Lali Khotenashvili4, Srdan Matic ${ }^{4}$ \\ IInfectious Diseases, AIDS and Clinical Immunology Research Center, Tbilisi, Georgia \\ ${ }^{2}$ Iv. Javakhisvhili Tbilisi State University. Faculty of Medicine, Tbilisi, Georgia \\ ${ }^{3}$ World Health Organization, Regional Office for Europe, Division of Sexually Transmitted Infections and HIVIAIDS, Tbilisi, Georgia \\ ${ }^{4}$ World Health Organization, Regional Office for Europe, Division of Sexually Transmitted Infections and HIV/AIDS, Copenhagen, Denmark
}

\begin{abstract}
SUMMARY
Introduction: HIV infection is the major public health, social and economic problem in Georgia. Although the HIV epidemic is in its nascent phase in the country, the potential risk for development of a wide spread HIV epidemic is very high.

The aim of this study is to evaluate the effectiveness of ARV treatment principles in Georgia, including treatment and monitoring methods.

Materials and Methods: The study included 985 people living with HIVIAIDS in Georgia registered at Infectious Disease, AIDS and Clinical Immunology Research Center since 2004. To ensure universal access to ARV therapy all HIVIAIDS individuals included in the study were investigated by special algorithm, all identified patients requiring ARV therapy were offered treatment and monitored during therapy on treatment effectiveness and side effects.

HIV-1 RNA in plasma was measured by quantitative Polymerase Chain Reaction. For determination of percentages and absolute count of Tlymphocyte subpopulations single-platform immunophenotyping technique using the Becton-Dickinson FACSCalibur flow cytometer was applied. For resistance testing TRUGENE HIV-1 Genotyping Kit with the OpenGene DNA Sequencing System (Siemens) was used. Reasons of treatment failure and mortality rate among ARV treated patients were analyzed.

Results and Conclusions: Treatment was offered to 398 HIVIAIDS patients. 397 patients started treatment, 1 patient refused. Out of 397 HIVIAIDS patients treated 21 patients discontinued, 54 patients died and 322 patients are currently on ARV treatment. Out of the treated patients 281 adults and 11 children are receiving first-line treatment, 27 adults and 2 children are on second-line treatment and 1 adult is receiving salvage regimen. Treatment failure was defined in 52 cases. Among them immunological failure was observed in 7 cases, clinical failure in 1 case and virologic failure in 44 cases. Prevalence of drug resistance among virologic failure cases accounted for $73 \%$ and inadequate adherence for $27 \%$ cases. Out of drug resistance cases 3\% has three-class drug resistance, $84 \%$ - two-class drug resistance and $13 \%$ found to be resistant to one class. In ARV naive patients the prevalence of drug resistance to any class was $4.33 \%$. The majority of death cases among ARV treated patients was due to non-AIDS related or incurable conditions, while deaths due to AIDS related conditions were mainly associated with delayed referral of patients in already advanced stage of disease. It's worth to mention that the highest number of death cases was due to liver failure in HIV/HCV and/or HBV co-infected patients.
\end{abstract}

Key words: HIVIAIDS, ARV treatment, drug resistance, fatality, Georgia

Address for correspondence: T. Tsertsvadze, Infectious Diseases, AIDS and Clinical Immunology Research Center 16 Al. Kazbegi Ave, Tbilisi, 0160 Georgia. E-mail: tengizt@gol.ge; aids@gol.ge

\section{INTRODUCTION}

Introduction of potent antiretroviral therapy (ART) in the mid 1990s can be regarded as a major breakthrough in HIV-related care. Antiretroviral drugs (ARV) have succeeded in delaying the onset of illness, decreasing mortality from AIDS, and improving the quality of life for people living with HIV/AIDS (PLHA) (1, 2). Since 2003 through the World Health Organization's (WHO) " 3 by 5 " strategy the access to ART is steadily increasing in resource limited countries (3). As a continuation of these efforts Universal Access (UA) initiative has been pledged by G8 Gleneagles Summit and the World Summit in 2005 to ensure UA to HIV/AIDS prevention, treatment, care and support by 2010 around the globe (4).
Georgia is an independent nation, formerly part of the Soviet Union. The first case of HIV infection in Georgia was reported in 1989. As of December 2007, a total of 1,472 HIV/AIDS cases were registered in the country, among them $76 \%$ were males. The estimated number of PLHA is around 3,000 as per Spectrum modeling. The annual number of newly reported HIV infections has risen each year. Of note, more than half of 1,472 registered HIV cases were reported in the past three years (2005-2007) (Fig 1).

The HIV epidemic in Georgia is mostly concentrated around the injecting drug users (IDU) accounting for $60 \%$ of the reported cumulative number. It's worth to mention that up to $68 \%$ of HIV infected individuals are co-infected with hepatitis viruses. Although the HIV epidemic is in its nascent phase, Georgia is believed to be at risk for an expanding epidemic due to the 


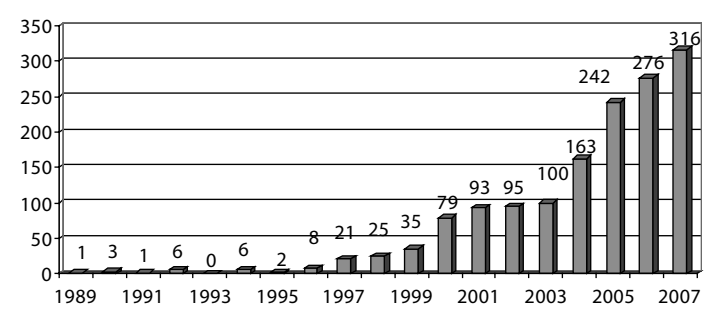

Fig 1. Number of new cases of HIV infection by year

widespread IDU; high rates of sexually transmitted infections (STI) and hepatitis B and C; and intense population movement between neighboring high-prevalence countries such as Ukraine and Russia.

The governement of Georgia has identified AIDS as a priority field back in the 1990s, and the progress over the last 5 years is commendable. Georgia was first among the former Soviet Union (FSU) countries to attain universal access to free-of-charge ART for all PLHA through the financial support of the Global Fund to fight AIDS, Tuberculosis and Malaria (GFATM) since 2004. This major step towards UA targets has been well acknowledged by the international community. According to the recent WHO, UNAIDS and UNICEF joint report, Georgia is among the 9 lowand middle-income countries that reached treatment coverage of at least 75 percent (5).

Diagnostics and treatment of AIDS patients in Georgia are provided at the highest attainable standards based on adaptation of the WHO, European, British HIV Association, DHHS of USA, IAS-USA Panel and other guidelines.

With the WHO and GFATM support:

1. National HIV/AIDS Treatment and Care Guidelines and Protocols have been developed in Georgia.

2. During 2004-2007, about 30 specialists completed longand/or short-term trainings in Georgia and abroad at the leading US and European HIV/AIDS Centers.

3. Several clinical workshops facilitated by leading European consultants/experts were conducted at the Infectious Diseases, AIDS and Clinical Immunology Research Center.

4. Modern principles of ARV treatment were implemented in Georgia.

Antiretroviral therapy itself is accompanied by side effects and the possibility of treatment failure and limitations of future therapeutic options should be well thought out before starting therapy (6-8). The CD4 T-cell count and the HIV RNA level serve as important predictors of disease progression and survival in untreated patients. It is well acknowledged fact that CD4 cell count is the primary marker for ARV treatment initiation, while the viral load serves as a secondary marker $(7,9-18)$. It is recommended that two nucleoside/nucleotide reverse transcriptase inhibitors (NRTIs) and one non-nucleoside reverse transcriptase inhibitor (NNRTI) be combined in the first-line ARV regimen.

A combination which backbone includes one of the Protease Inhibitors (PI), known for higher genetic barriers, is recommended for use in second-line ARV treatment. Second-line ARV treatment is recommended only in case of proven treatment failure. Initiating second-line treatment has to be accompanied by strong efforts to reassess and support adherence. In case of confirmed second- line ARV treatment failure (using virological, immunological or clinical criteria), a salvage regimen is considered.

The aim of this study is to evaluate effectiveness of ARV treatment principles in Georgia, including treatment and monitoring methods.

\section{MATERIALS AND METHODS}

\section{Subjects}

The investigation was conducted at the Infectious Diseases, AIDS \& Clinical Immunology Research Center (IDACIRC) of Georgia. Study included all HIV/AIDS patients, who have been registered at IDACIRC and available in Georgia since 2004. Out of cumulative 1,472 HIV/AIDS cases registered at the IDACIRC, 321 died and 166 were out of country. Therefore, 985 patients were included in the study, 694 men and 156 women. All study subjects were investigated by special algorithm to evaluate the need of ARV treatment. Patients were aged from 3 months to 64 years. $68 \%$ of study subject were co-infected with HCV, HBV or both.

\section{Study Design}

To ensure universal access to ARV therapy all 985 HIV/AIDS individuals included in the study were requested to submit to following examinations:

1. Investigation of all HIV infected persons by special algorithm:

- Physical examination.

- Complete blood count.

- Immunological investigation (including CD4 count).

- Viral Load (HIV RNA by quantitative PCR).

- Clinical chemistry (wider range).

- Ultrasound, X-ray, CT-scan.

Monitoring of CD4 cell count, HIV RNA viral load dynamic and appearance of opportunistic infections were scheduled in every 3 months.

2. Identification of all patients requiring ARV therapy according to special criteria described in National Guidelines on HIV/AIDS treatment:

- Symptomatic HIV infection (AIDS or severe symptoms).

- CD4 count $<200 / \mathrm{mm}^{3}$.

- if CD4 is $201-350 / \mathrm{mm}^{3}$, decision is made upon CD4 decline rate, viral load, presence of hepatitis co-infection.

3. Offering ARV therapy to all eligible patients requiring treatment.

At present in Georgia the National Guidelines recommend as a first-line ARV regimen combination of two nucleoside/nucleotide reverse transcriptase inhibitors (NRTIs) and one non-nucleoside reverse transcriptase inhibitor (NNRTI). Second-line ARV regimen includes one of the Protease Inhibitors (PIs) boosted with Ritonavir and two NRTIs. Salvage regimen includes Fusion Inhibitor Enfurvitide (ENF) and the new PIs - Tipranavir (TPV) or Darunavir (Prezista). Another option is a combination of two PIs, except TPV, which is not to be combined with any other PIs. It's worth to mention that during selection of ARV regimen 
following factors has been taken into account: hepatotoxicity of non-nucleoside reverse transcriptase inhibitors (NNRTIs) (such as with Nevirapine (NVP) and presence of hepatic injury, use of opioid substitution therapy (pharmacokinetic interaction between NNRTIs and methadone or buprenorphine), coexistent medical or psychiatric conditions and etc.

4. Monitoring of all patients receiving ARV treatment was done at baseline, after 8 weeks of treatment initiation and then in every 3 months. Clinical symptoms, CD4 T-cell count, HIV RNA level, side-effects and adherence were monitored during the follow-up visits. Possible side effects of particular drugs could require more frequent monitoring and this was decided on individual basis. Treatment failure was defined according to the National Protocols:

- Virologic failure:

a) $\mathrm{VL}>400 \mathrm{c} / \mathrm{ml}$ at 24 weeks,

b) $\mathrm{VL}>50 \mathrm{c} / \mathrm{ml}$ at 48 weeks,

c) after viral suppression VL rebound to $>400 \mathrm{c} / \mathrm{ml}$ at two occasions in 4 to 8 weeks.

- Immunological failure:

a) Increase in CD4 cell count $\leq 50$ cells $/ \mathrm{mm} 3$ during a first year,

b) $>50 \%$ fall from on therapy CD4 peak level.

- Clinical failure:

a) Occurrence of new opportunistic infections (OI),

b) Recurrence of prior OI,

c) Onset of WHO stage III conditions.

In case of severe opportunistic infections like TB, pneumocystic pneumonia etc., treatment was deferred for few days or weeks until disease stabilization, to avoid overlapping toxicity of drugs and possibility of Immune Reconstitution Syndrome (IRIS). However, this was done with caution in patient with CD4 count below the 50 cells $/ \mathrm{mm}^{3}$, where ARV treatment was initiated as soon as possible.

In case of treatment failure adherence was reassessed and genotypic resistance test was performed to determine whether failure was due to HIV drug resistance or other causes (e.g., inadequate adherence, drug side effects) and consequently second-line or salvage ARV regimen was initiated.

To ensure maximal adherence before initiation of ARV treatment all patients were educated and prepared for adherence. Following methods were used for adherence assessment: Offering patients specially designed table-sheet for reporting daily (morning and evening) medication intake; Offering patients specially designed questionnaire to identify reasons for missing doses; Asking patient to describe medication intake during the preceding three days; Counting pills remaining in box/bottles with consideration of dosage provided at patients last visit; Identification of pills by patients that are in their regimen by shape and colour.

\section{METHODS}

Detection of HIV antibodies was performed by ELISA (third or fourth generation) with further confirmation by Western Blot Assay.

HIV-1 RNA in plasma was measured by commercially available quantitative Polymerase Chain Reaction (PCR) method
(AMPLICOR HIV-1 MONITOR Test, version 1.5, Hoffmann-La Roche, Inc.) and run according to the protocol of the manufacturer. Since 2006 The COBAS TaqMan HIV-1 test (Real time PCR) has been used for quantification of HIV-1 RNA in Hunam EDTA plasma, using the High Pure System Viral Nucleic Acid Kit for manual specimen preparation and the COBAS TaqMan 48 Analyzer for automated amplification and detection. The test can quantify HIV-1 RNA over range of 40-10,000,000 copies $/ \mathrm{ml}$.

For determination of percentages and absolute count of T-lymphocyte subpopulations (T-helpers, T-cytotoxic,) specific markers were measured according to the standard by CDC recommended single-platform immunophenotyping technique using the BectonDickinson FACSCalibur flow cytometer. Complex of following monoclonal antibodies was used CD3FITC/ CD8PE/ CD45PerCP/ CD4APC. In brief this method consists of steps as follows: 1. Incubation of sample with relevant monoclonal antibodies in True Count tubes; 2. Lysing of erythrocytes using lysing solution; 3. Incubation and following analyzing on flow cytometer using appropriate software. Data for a minimum of 5,000 lymphocytes were acquired for each sample.

For resistance testing TRUGENE HIV-1 Genotyping Kit with the OpenGene DNA Sequencing System (Siemens) was used. Resistance testing involves the following steps:

1. HIV-1 RNA extraction; 2. Reverse Transcription and PCR Amplification of the target sequence in the pol gene; 3. CLIP/ Sequencing Reaction; 4. Gel electrophoresis of CLIP reaction products; 5. Sequence Analysis. The Guidelines ${ }^{\mathrm{TM}}$ software of the TruGene system was used to interpret the genotype for drug resistance and producing a clinical report. The sequences obtained were also analyzed using the software available http://hivdb. stanford.edu/.

\section{RESULTS}

Of 985 patients investigated 398 were in need of ARVs and treatment was offered to all of them. Out of 398 patients 295 were symptomatic and 103 asymptomatic. Asymptomatic patients were offered treatment based on immunological and virological criteria. 397 patients started treatment, 1 patient refused. Among them 380 were adults and 17 children under age of 15 . Out of treated patients 21 self-discontinued treatment, 54 patients died and 322 patients (309 adult and 13 children) remained on ARV treatment. Out of treated patients 281 adults and 11 children are receiving first-line treatment and 27 adults and 2 children are already on second-line treatment, 1 adult is receiving salvage regimen.

Distribution of various first-line and second-line ARV regimens among adult patients is presented in Table 1 and Table 2 accordingly.

One patient is receiving salvage regimen including Darunavir and Enfuvirtide.

Reasons of switching ARV from first to second-line were analyzed. Treatment failure was reported in 52 cases. Among them immunological failure was observed in 7 cases, clinical failure in 1 case and virologic failure in 44 cases. In all virologic failure cases adherence was reassessed and genotypic resistance test was performed while patient was still on treatment. Of 44 cases in $32(73 \%)$ failure was attributable to drug resistance. Of 32 patients with HIV drug resistance 1 patient (3\%) has three-class 
Table 1. Distribution of patients receiving first-line ARV treatment

\begin{tabular}{|l|c|c|}
\hline First-line ARV regimens & $\begin{array}{c}\text { Number of } \\
\text { adults }\end{array}$ & $\begin{array}{c}\text { Number of } \\
\text { children }\end{array}$ \\
\hline ZDV + 3TC + EFV & 168 & 4 \\
\hline ZDV + 3TC + NVP & 36 & 3 \\
\hline TDF + FTC + EFV & 16 & - \\
\hline TDF + FTC + NVP & 3 & - \\
\hline$A B C+3 T C+E F V$ & 55 & 2 \\
\hline$A B C+3 T C+N V P$ & 3 & 2 \\
\hline
\end{tabular}

Table 2. Distribution of patients receiving second-line ARV treatment

\begin{tabular}{|l|c|c|}
\hline Second-line ARV regimens & $\begin{array}{c}\text { Number of } \\
\text { adults }\end{array}$ & $\begin{array}{c}\text { Number of } \\
\text { children }\end{array}$ \\
\hline $\begin{array}{l}\text { LPV/r (SQV/r or FPV/r) + TDF } \\
+A B C\end{array}$ & 6 & - \\
\hline $\begin{array}{l}\mathrm{LPV} / \mathrm{r}(\mathrm{FPV} / \mathrm{r} \text { or ATV/r) + ddl } \\
+\mathrm{ABC}\end{array}$ & 18 & 2 \\
\hline $\mathrm{LPV} / \mathrm{r}+\mathrm{TDF}+(\mathrm{ZDV}+3 \mathrm{TTC})$ & 3 & - \\
\hline
\end{tabular}

drug resistance (NRTI, NNRTI and PI); 27 patients (84\%) had two-class drug resistance, 26 patients to NRTI and NNRTI drugs and 1 patient - to NRTIs and PIs and 4 patients $(13 \%)$ found to be resistant to one class: NNRTIs. In 12 cases (27\%) virologic failure due to non-adherence was found that was confirmed by detectable viremia, without resistance mutations. Out of these 12 cases 2 patients discontinued treatment and in 10 cases adherence was ensured. Out of 32 cases with drug resistance all patients were switched to second-line regimen. Out of them currently 27 adult patients and 2 children were switched and remained on secondline ARVs, 1 patient discontinued second line regimen and 1 was switched to salvage regimen, 1 patient died.

Common NRTI mutations reported in treatment experienced patients included: the signature Lamivudine mutation M184V has been detected in 26 subjects; 1 subject was found to be resistant to Zalcitabine (ddC) and Tenfovir (TDF) due to K65R mutation; 2 subjects had intermediate resistance to Zidovudine (AZT) and possibly to Stavudine (d4T) due to T215Y/F+K219Q; 1 subject had possible resistance to Zidovudine (AZT) and Stavudine (d4T) due to $\mathrm{T} 215 \mathrm{Y} / \mathrm{F}$, which also limits the effectiveness of Abacavir $(\mathrm{ABC})$, Didanosine (ddI) and particularly Tenofovir (TDF).

As for NNRTI mutations: 11 subjects had K103N mutation causing resistance to Nevirapine (NVP), Delaviridine (DLV) and Efavirenz (EFV); 7 subjects were resistant to Nevirapine (NVP) and Efavirenz (EFV) due to G190S mutation with presence K101E mutation in two cases; 3 subjects were resistant to Nevirapine (NVP) and Delaviridine (DLV) due to Y181C mutation, one subject had resistance to same drugs caused by V106I mutation; In addition, M230, A98G, V179D/E and V108I mutations were detected in these subjects, which cause resistance to each of the available NNRTIs.

Resistance to protease inhibitors have been found in 2 subjects due to D30N and M46I + N88S mutations.
It is worth to mention that in order to evaluate prevalence of drug resistance in ARV naive patients and to further optimize ARV in Georgia, we have studied newly diagnosed 184 ARV naive patients for drug resistance. Following prevalence of resistance among naive subjects was found:

- $4.33 \%$ had resistance to any ARV class.

- $3.26 \%$ were resistant to 1 class.

- $1 \%$ was resistant to 2 class.

- No 3 class resistance was found.

Among 184 ARV-naive patients: 2 patients showed to be resistant to all available NNRTIs due to K103N mutation; 2 patients were resistant to Nevirapine due to K101E; 4 patients had M46I mutation indicating resistance to Indinavir (IDV) and Amprenavir (APV)/Fosamprenavir (FPV); 2 patients had M184V mutation indicating resistance to $3 \mathrm{TC}$. During the study period out of investigated $184 \mathrm{ARV}$ naive patients no one started ARV therapy. Their baseline resistance pattern will be taken into account while prescribing ARV regimen in future.

Fatality rate and reasons of death was studied among treated patients. Overall fatality rate of ARV treated patients was $14 \%$ (54 patients out of 397). Of died cases in 12 was their death attributable to the late stage of HIV/AIDS complicated with lifethreatening diseases, 13 cases were due to incurable malignancies and 29 were due to non-AIDS related death. Table 3 summarizes reasons of deaths among patients receiving ARV. It is worth to mention that the highest number of deaths was due to liver failure in $\mathrm{HIV} / \mathrm{HCV}$ and/or HBV co-infections. This fact once more emphasizes that co-infection with hepatitis viruses is of major concern in HIV patients.

Table 3. Causes of death among patients receiving ARV

\begin{tabular}{|l|c|}
\hline $\begin{array}{l}\text { Reasons of death among patients } \\
\text { receiving ARV }\end{array}$ & Number of deaths \\
\hline AIDS related (total) & 12 \\
\hline Toxoplasmic encephalitis & 1 \\
\hline Cryptococcal meningitis & 2 \\
\hline Tuberculous meningoencephalitis & 3 \\
\hline Wasting syndrome & 1 \\
\hline Meningoencephalitis of unknown etiology & 2 \\
\hline Lung tuberculosis & 3 \\
\hline Cancers (total) & 13 \\
\hline Lymphoma & 4 \\
\hline Kaposi's Sarcoma & 2 \\
\hline Colon cancer & 3 \\
\hline Lung cancer, respiratory failure & 4 \\
\hline Non-AIDS related & 29 \\
\hline Myocardial infarction & 3 \\
\hline Hemorrhagic stroke & 3 \\
\hline Cerebral trauma & 2 \\
\hline Acute renal failure & 2 \\
\hline Liver failure & 19 \\
\hline Total & 54 \\
\hline
\end{tabular}




\section{DISCUSSION}

In the presented study we aimed to evaluate effectiveness of ARV treatment principles in Georgia, including treatment and monitoring methods.

Study included 985 PLHA in Georgia registered at Infectious Disease, AIDS and Clinical Immunology Research Center and available in the country since 2004 .

High standards of diagnostics and treatment of AIDS patients in Georgia is achieved by adaptation of the WHO and Western guidelines with WHO and GFATM support, also by wide-scale long- and short-term trainings of about 30 specialists completed in and outside the country at the leading US and European HIV/ AIDS Centers.

Universal access to ARV was achieved by investigation of all HIV infected persons by special algorithm, identification all patients requiring ARV therapy, offering ARV therapy to all patients requiring treatment and monitoring of all patients receiving ARV treatment.

Reasons of treatment failure were analyzed by assessing the reasons of switching ARV regimen and fatality rate among ARV treated patients. Treatment failure was reported in 52 cases. Among them immunological failure was observed in 7 cases, clinical failure in 1 case and virologic failure in 44 cases. Virologic failure was observed in 44 cases (11\% of all treated patients). Out of 44 cases in 32 cases (73\%) failure was attributable to drug resistance. Out of 32 patients with HIV drug resistance 1 patient (3\%) has three-class drug resistance, 27 patients $(84 \%)$ had two-class drug resistance and 4 patients $(13 \%)$ found to be resistant to one class. In 12 cases (27\%) virologic failure due to non-adequate adherence was found and confirmed by detectable viremia, without resistance mutations. In addition, prevalence of HIV drug resistance in ARV drug naive patients was found to be $4.33 \%$. Our data are comparable with the data of other resourcelimited countries with increasing access of ARV therapy (19-24). These data suggest that the incidence of transmitted drug resistance is still low but is rising along with increasing access to ARV. On the other side current estimates indicate that the prevalence of transmitted drug resistance is highest in regions and populations with long-established use of antiretroviral therapy (25-33).

All these data will assist us to further optimize resistance testing strategy and, in general, ARV treatment in Georgia.

Overall fatality rate of ARV treated patients was 14\% (54 patients out of 397). It is interesting that death cases were attributable to the late stage of HIV/AIDS complicated with life-threatening diseases, incurable malignancies and there were also non-AIDS related death. Among these patients CD4 cell counts were low and plasma viral loads were higher at baseline. Data from very large cohort studies have shown that both baseline (34) and current (35) low CD4 cell counts and baseline high plasma viral load are associated with a higher risk of death. Highest number of death cases was due to liver failure in HIV/HCV and/or HBV co-infected patients. Several studies have highlighted the increasing importance of liver-related disease as a cause of death in HIVinfected patients in pre-, early, and late $\operatorname{ARV}(36,37)$. In patients coinfected with viral hepatitis $\mathrm{B}$ or $\mathrm{C}$ increasing fatality rate from liver-related diseases was shown (38). Specific analysis of the EuroSIDA cohort found a decreasing rate of fatality from liverrelated disease across Europe after wide use of ARV. However, after adjustment for the current CD4 cell count, and consequent increase in CD4 cell counts in patients taking ARV, there was a significant increase over time in death rates from liver-related disease. In addition, in patients with similar CD4 cell counts, longer exposure to ART was associated with an increased death rate from liver-related disease. This may be explained by direct liver toxicity of antiretrovirals, progression of liver disease due to hepatitis $\mathrm{B}$ virus or hepatitis $\mathrm{C}$ virus over time as patients survive longer, or some other factor (39). Despite the changing trend of fatality rate from liver-related diseases in HIV infected patients on ARV over time (39), our data suggest that liver-related deaths are pointing out to the problem, which calls for urgent action against hepatitis viruses co-infection in HIV patients on a national level.

Conflict of interest: there are no financial or any other type of conflict of interests with regards to analyses presented in our paper ("Experience of Antiretroviral treatment in Georgia").

\section{REFERENCES}

1. Palella FJ Jr, Delaney KM, Moorman AC, Loveless MO, Fuhrer J, Satten GA, et al; HIV Outpatient Study Investigators. Declining morbidity and mortality among patients with advanced human immunodeficiency virus infection. N Engl J Med. 1998 Mar 26;338(13):853-60.

2. Mocroft A, Ledergerber B, Katlama C, Kirk O, Reiss P, d'Arminio Monforte A, et al; EuroSIDA study group. Decline in the AIDS and death rates in the EuroSIDA study: an observational study. Lancet. 2003 Jul 5;362(9377):22-9.

3. World Health Organization; UNAIDS. Progress on global access to HIV antiretroviral therapy. A report on "3 by 5" and beyond. Geneva: WHO; 2006.

4. United Nations [homepage on the Internet]. 2005 World Summit Outcome. General Assembly 60/1. 24 October 2005 [cited 2007 Dec 17]. Available from: http://unpan1.un.org/intradoc/groups/public/documents/UN/UNPAN021752.pdf.

5. World Health Organization; UNAIDS; UNICEF. Towards universal access: scaling up priority HIV/AIDS interventions in the health sector. Progress report 2008. Geneva: WHO; 2008.

6. Yeni PG, Hammer SM, Carpenter CC, Cooper DA, Fischl MA, Gatell JM, et al. Antiretroviral treatment for adult HIV infection in 2002: updated recommendations of the International AIDS Society -USA Panel. JAMA. 2002 Jul 10;288(2):222-35. Erratum in: JAMA. 2003 Jan-Feb;11(1):32.

7. The EACS Euroguidelines Group. European guidelines for the clinical management and treatment of HIV infected adults in Europe. AIDS. 2003 Jun;17 Suppl 2:S3-26.

8. World Health Organization. Patient evaluation and antiretroviral treatment for adults and adolescents. Clinical Protocol for the WHO European Region. Copenhagen: WHO Regional Office for Europe; 2006.

9. British HIV Association [homepage on the Internet]. British HIV Association guidelines for the treatment of HIV-infected adults with antiretroviral therapy, 2003 [cited 2007 Dec 17]. Available from: http://www.bhiva. org/files/file1001306.pdf

10. United States Department of Health and Human Services. Guidelines for the use of antiretroviral agents in HIV-1 infected adults and adolescents. Bethesda: DHSS, 2004.

11. Salzberger B, Marcus U, Vielhaber B, Arasteh K, Gölz J, Brockmeyer $\mathrm{NH}$, et al. German-Austrian recommendations for the antiretroviral therapy of HIV-infections (status May 2004). Eur J Med Res. 2004 Nov 29;9(11):491-504.

12. Egger M, May M, Chêne G, Phillips AN, Ledergerber B, Dabis F, et al; ART Cohort Collaboration. Prognosis of HIV-1-infected patients starting highly active antiretroviral therapy: a collaborative analysis of prospective studies. Lancet. 2002 Jul 13;360(9327):119-29. Erratum in: Lancet 2002 Oct 12;360(9340):1178.

13. Phillips AN, Pradier C, Lazzarin A, Clotet B, Goebel FD, Hermans P, et al; EuroSIDA Study Group. Viral load outcome of non-nucleoside reverse transcriptase inhibitor regimens for 2203 mainly antiretroviralexperienced patients. AIDS. 2001 Dec 7; 15(18):2385-95. 
14. Sterling TR, Chaisson RE, Keruly J, Moore RD. Improved outcomes with earlier initiation of highly active antiretroviral therapy among human immunodeficiency virus-infected patients who achieve durable virologic suppression: longer follow-up of an observational cohort study. J Infect Dis. 2003 Dec 1;188(11):1659-65.

15. Opravil M, Ledergerber B, Furrer H, Hirschel B, Imhof A, Gallant S, et al; Swiss HIV Cohort Study. Clinical efficacy of early initiation of HAART in patients with asymptomatic HIV infection and CD4 cell count $>350 \mathrm{x}$ 10(6) /1. AIDS. 2002 Jul 5;16(10):1371-81.

16. Gras L, van Sighem A, Fraser C, Griffin J, Lange J, Miedema F, et al; ATHENA Cohort Study Group. Predictors of changes in CD4 cell count seven years after starting HAART. In: 13th Annual Conference on Retroviruses and Opportunistic Infections; 2006 Feb 5-8; Denver, USA. Alexandria: CROI; 2006. abstract 530.

17. Palella FJ Jr, Deloria-Knoll M, Chmiel JS, Moorman AC, Wood KC, Greenberg AE, et al; HIV Outpatient Study Investigators. Survival benefit of initiating antiretroviral therapy in HIV-infected persons in different CD4+ cell strata. Ann Intern Med. 2003 Apr 15;138(8):620-6.

18. Keruly J, Moore R. Increases in CD4 cell count to five years in persons with sustained virologic suppression. In: 13th Annual Conference on Retroviruses and Opportunistic Infections; 2006 Feb 5-8; Denver, USA. Alexandria: CROI; 2006. abstract 529.

19. Rios M, Vazquez de Parga E, Pérez-Alverez L, et al. Analysis of resistance associated-mutations in patients infected with HIV-1 of subtype B and non-B from Chile. In The proceedings of 4th European HIV Drug Resistance Workshop; 2006 Mar 29-31; Monte Carlo. Abstract 27.

20. Agwale SM, Zeh C, Paxinos E, Odama L, Pienazek D, Wambebe C, et al. Genotypic and phenotypic analyses of human immunodeficiency virus type 1 in antiretroviral drug-naive Nigerian patients. AIDS Res Hum Retroviruses. 2006 Jan;22(1):22-6.

21. Tee KK, Kamarulzaman A, Ng KP. Low prevalence of genotypic drug resistance mutations among antiretroviral-naive HIV type 1 patients in Malaysia. AIDS Res Hum Retroviruses. 2006 Feb 1;22(2):121-4.

22. Vergne L, Diagbouga S, Kouanfack C, Aghokeng A, Butel C, Laurent C, et al. HIV-1 drug-resistance mutations among newly diagnosed patients before scaling-up programmes in Burkina Faso and Cameroon. Antivir Ther. 2006;11(5):575-9.

23. Rodrigues R, Scherer LC, Oliveira CM, Franco HM, Sperhacke RD, Ferreira JL, et al. Low prevalence of primary antiretroviral resistance mutations and predominance of HIV-1 clade $\mathrm{C}$ at polymerase gene in newly diagnosed individuals from south Brazil. Virus Res. 2006 Mar;116(1-2):201-7.

24. Lama JR, Sanchez J, Suarez L, Caballero P, Laguna A, Sanchez JL, et al. Linking HIV and antiretroviral drug resistance surveillance in Peru: a model for a third-generation HIV sentinel surveillance. J Acquir Immune Defic Syndr. 2006 Aug 1;42(4):501-5.

25. Jørgensen LB, Gerstoft J, Mathiasen L, et al. Low prevalence of transmitted HIV-1 drug resistance in newly diagnosed HIV-1 patients in Denmark from 2000-2004. In The Proceedings of 4th European HIV Drug Resistance Workshop; 2006 Mar 29-31; Monte Carlo. Abstract 22.

26. Fox J, Hill S, Kaye S, Dustan S, McClure M, Fidler S, et al. Prevalence of primary genotypic resistance in a UK centre: comparison of primary HIV-1 and newly diagnosed treatment-naive individuals. AIDS. 2007 Jan $11 ; 21(2): 237-9$
27. Wensing AM, van de Vijver DA, Bentum PH, et al. Truly representative surveillance of HIV baseline drug resistance and subtypes in the Netherlands. In The Proceedings of 3rd European HIV Drug Resistance Workshop; 2005 Apr 4-7; Athens. Abstract 7.

28. Booth CL, Garcia-Diaz AM, Youle M, Johnson MA, Phillips A, Geretti AM. Prevalence and predictors of antiretroviral drug resistance in newly diagnosed HIV-1 infection. J Antimicrob Chemother. 2007 Mar;59(3):517-24.

29. Yerly S, von Wyl V, Boni J, et al. Transmission of HIV-1 drug resistance in Switzerland: a 10-year molecular epidemiology survey. Antivir Ther. 2006;11(5):S118.

30. Routy JP, de Edwardes D, Rouleau D, et al. Influence of patient characteristics, year of infection, CD4 cell count and viral load on the presence of primary HIV-1 drug resistance in recently infected patients. Antivir Ther. 2005;10 Suppl 1:S133.

31. Truong HM, Klausner JD, Hecht FM, et al. Reduced levels of primary resistance to nRTIs in San Francisco is discernable using two independent sentinel populations. Antivir Ther. 2006;11(5):S115.

32. Jayaraman GC, Archibald CP, Kim J, Rekart ML, Singh AE, Harmen S, et al. A population-based approach to determine the prevalence of transmitted drug-resistant HIV among recent versus established HIV infections: results from the Canadian HIV strain and drug resistance surveillance program. J Acquir Immune Defic Syndr. 2006 May;42(1):86-90.

33. Wensing AMJ, Vercauteren J, van de Vijver DA, et al. Transmission of drug-resistance in Europe is characterized by single mutations and revertants. Antivir Ther. 2006;11(5):S111.

34. Sterne JA, Hernán MA, Ledergerber B, Tilling K, Weber R, Sendi P, et al. Long-term effectiveness of potent antiretroviral therapy in preventing AIDS and death: a prospective cohort study. Lancet. 2005 Jul 30-Aug 5;366(9483):378-84.

35. Weber R, Friis-Møller N, Sabin C, et al. HIV and non-HIV-related deaths and their relationship to immunodeficiency: In The proceedings of 12th Conference on Retroviruses and Opportunistic Infections; 2005 Feb 2225; Boston. Abstract 595

36. Louie JK, Hsu LC, Osmond DH, Katz MH, Schwarcz SK. Trends in causes of death among persons with acquired immunodeficiency syndrome in the era of highly active antiretroviral therapy, San Francisco 1994-1998. J Infect Dis. 2002 Oct 1;186(7):1023-7.

37. Crum NF, Riffenburgh RH, Wegner S, Agan BK, Tasker SA, Spooner $\mathrm{KM}$, et al. Comparisons of causes of death and mortality rates among HIV-infected persons: analysis of the pre-, early, and late HAART (highly active antiretroviral therapy) eras. J Acquir Immune Defic Syndr. 2006 Feb 1;41(2):194-200

38. Rockstroh J, Konopnicki D, Soriano V, Kirk O, Antunes F, Knysz B, et al; EuroSIDA study group. Hepatitis B and hepatitis C in the EuroSIDA cohort: prevalence and effect on mortality, AIDS progression and response to HAART. In The proceedings of 11 th Conference on Retroviruses and Opportunistic Infections; 2004 Feb 8-11; San Francisco. Abstract 799.

39. Mocroft A, Soriano V, Rockstroh J, Reiss P, Kirk O, de Wit S, et al; EuroSIDA Study Group. Is there evidence for an increase in the death rate from liver-related disease in patients with HIV? AIDS. 2005 Dec 2;19(18):2117-25 